\title{
Detection and Enumeration of the Commonest Stool Parasites Seen in a Tertiary Care Center in South India
}

\author{
Vani Chandrashekar \\ Department of Hematology and Clinical Pathology, Apollo Hospitals, 21 Greams Lane, Off Greams Road, Chennai, Tamil Nadu, India \\ Correspondence should be addressed to Vani Chandrashekar; drvani001@gmail.com
}

Received 8 May 2013; Accepted 10 June 2013

Academic Editors: F. Fouque and Z.-G. Han

Copyright (c) 2013 Vani Chandrashekar. This is an open access article distributed under the Creative Commons Attribution License, which permits unrestricted use, distribution, and reproduction in any medium, provided the original work is properly cited.

\begin{abstract}
The aim of this study was to identify common stool parasites in patients attending a tertiary care centre in South India. We evaluated 2355 stool samples and parasites were detected in $7.9 \%$ of samples. $41.1 \%$ of our patients were in the $45-58$-year age group. Protozoal infections were the commonest seen in $7.8 \%$ of samples. Entamoeba histolytica was the commonest protozoa (4.6\%) followed by Entamoeba coli (1.2\%) and Giardia (0.8\%). Entamoeba histolytica and Entamoeba coli were together seen in $0.63 \%$, and they were the commonest organisms seen in samples with multiple-organism infection. Both were equally detected in diarrheal samples.
\end{abstract}

\section{Introduction}

Approximately, $60 \%$ of the world's population is infected with intestinal parasites [1]. Commonest parasites seen worldwide are Ascaris (20\%), Ancylostoma (18\%), Trichuris (10\%), and Entamoeba histolytica (10\%) [2].

The incidence of protozoa and helminthes in stool varies with geographical location. In a study in food handlers in Iran, it was seen that Giardia was the commonest pathogen (4.5\%). Other pathogens were Hymenolepis nana (1.29\%), Entamoeba histolytica (1.39\%), and Ascaris lumbricoides (0.57\%). Entamoeba coli was seen in 9.1\%, Iodamoeba in $5 \%$ and Blastocystis hominis in $11.3 \%$ [3]. In an Ethiopian study, prevalence of hookworm was highest (60.2\%), followed by Schistosoma mansoni (21.2\%), Trichuris (14.7\%), Taenia species (13.9\%), Entamoeba histolytica (12.7\%), Ascaris lumbricoides (6.2\%), Giardia (6.2\%), and Strongyloides (5.8\%) [4]. Eligail et al. reported that the commonest stool parasites reported in Riyadh, Saudi Arabia, were E. coli (4.08\%), Iodamoeba (1.79\%), Endolimax nana (1.75\%), Ascaris (0.67\%), Giardia (0.67\%), Chilomastix (0.41\%), Hookworm (0.36\%), E. histolytica (0.14\%), Hymenolepis nana (0.10\%), Strongyloides (0.07\%), Trichomonas (0.06\%), Schistosoma mansoni $(0.04 \%)$, Balantidium (0.04\%), Enterobius vermicularis (0.02\%), and Taenia species (0.01\%) [5]. In western Nepal,
Giardia was the commonest (13.2\%) followed by Ascaris (2.1\%) and Entamoeba histolytica (1.7\%) [6].

Study by Sehegal et al. found that prevalence rate of protozoa infection was $81.2 \%$ whereas that of helminthes was $18.8 \%$ [7]. They found that the commonest pathogen in children and pregnant women was Giardia (21.4\% and $6.9 \%$, resp.) followed by E. histolytica (5.3\% and $4.6 \%)$. In a recent study by Srihari et al., it was found that $E$. histolytica was the commonest parasite $(43.8 \%)$ followed by Cryptosporidium parvum (29.8\%) and Giardia (10.53\%) [8]. In Marothi and Singh's study, E. histolytica was the commonest (10.5\%) followed by Giardia (3.9\%), and among helminthes, Ascaris was the commonest (2.8\%) [9].

The aim of this study was to identify common stool parasites in patients attending a tertiary care center in South India.

\section{Materials and Methods}

We examined stool samples from 2355 patients who underwent a regular health checkup. Stool was collected in wide mouthed, clean containers and transported to the laboratory. The samples were grossly examined for color, consistency, and presence of proglottids. The samples were microscopically examined within 1 hour of collection. Saline and iodine wet mounts were prepared by adding a drop of saline and 


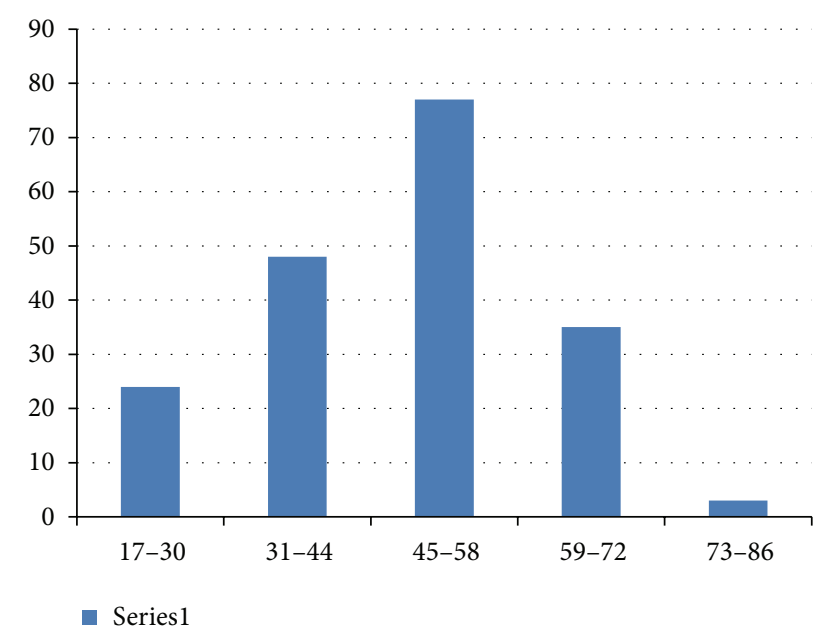

FIGURE 1: Frequency of parasite detection in different age groups (age group $-x$-axis and frequency $-y$-axis).

lugols iodine to clean glass slides and then mixed with a small amount of stool. A coverslip was placed and the slide was visualized microscopically first at low power to detect trophozoites and eggs and then at higher power for morphological details. If parasites were detected on wet mount, a dried smear was prepared and fixed with $70 \%$ alcohol. This was then stained with trichrome stain for 14 minutes followed by destaining in $70 \%$ ethanol and acid alcohol (90\% ethanol and acetic acid). They were then cleared with xylene, mounted, and allowed to dry. Trichrome-stained smears were visualized in high power and oil immersion lens. Stool concentration with ethyl acetate was carried out when there was clinical suspicion of parasite infection.

\section{Observations}

Among 2355 stool samples, we detected parasites in 187 (7.9\%) patients. Of these, protozoa were detected in $185(7.8 \%)$ and helminthes in $2(0.08 \%)$ samples. There were 95 males and 92 females with age ranging from 18 to 82 years. Of these, 77 (41.1\%) were in the 45-58 year age group (Figure 1).

Infection by single organism was seen in 166 (7.04\%) patients. Double and multiple organism infection was seen in only $21(0.89 \%)$ patients.

Table 1 lists the organisms we encountered in stool samples containing single organism only. The commonest in this group is Entamoeba histolytica (4.67\%).

Table 2 lists the organisms we detected in stool samples containing multiple organisms. The commonest combination of organisms we saw was Entamoeba histolytica and Entamoeba coli $(0.63 \%)$.

We received 29 diarrheal samples. Table 3 lists the frequency of detection of various organisms in diarrheal and nondiarrheal samples. It is evident from the table that $E$. histolytica, E. coli, and Giardia lamblia were detected in both diarrheal and nondiarrheal samples. However, the commonest organism seen in diarrheal samples was E. coli (39.2\%) and E. histolytica (39.2\%).

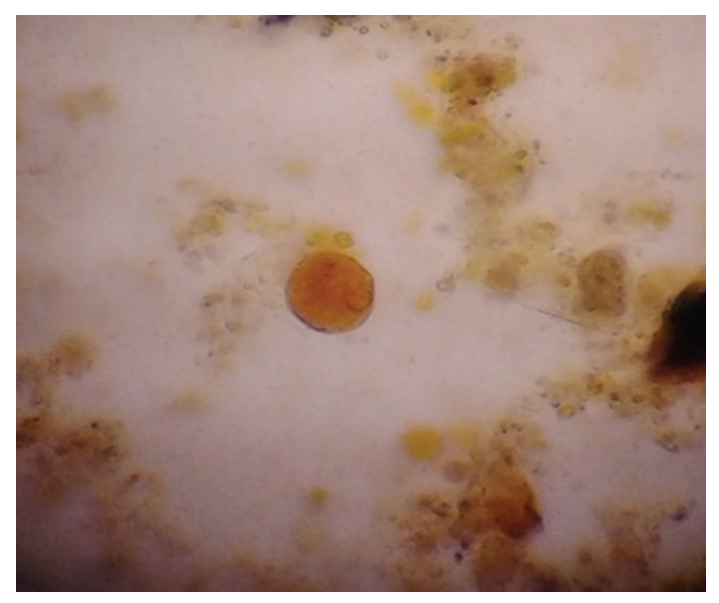

Figure 2: EH cyst (iodine).

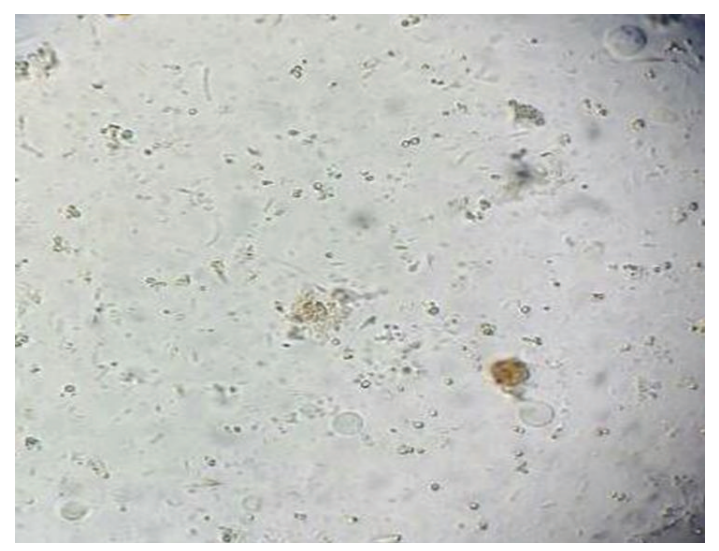

FIgURE 3: EH cyst (saline).

TABLE 1: Frequency of infection by single organism.

\begin{tabular}{lc}
\hline Organism & Number \\
\hline E. histolytica & $110(4.67 \%)$ \\
E. coli & $29(1.23 \%)$ \\
G. lamblia & $20(0.84 \%)$ \\
C. mesnili & $1(0.04 \%)$ \\
I. bütschlii & $3(0.12 \%)$ \\
T. hominis & $1(0.04 \%)$ \\
E. vermicularis & $1(0.04 \%)$ \\
S. stercoralis & $1(0.04 \%)$ \\
\hline
\end{tabular}

\section{Identification of Organisms (Figures 2-12)}

E. histolytica. In saline mount (Figure 3) smears cysts were visualized as small spherical structures which were difficult to differentiate from leucocytes. The nucleus was not visualized. In iodine mount (Figure 2), some of the cysts showed small nucleus against a cytoplasmic brown background. In trichrome stain (Figure 5) the cytoplasm appeared granular and greenish with prominent red nuclei. In our samples most of the cysts were uninucleate with rare bi- and quadrinucleate forms. Chromatoid bodies (Figure 6) stained red 


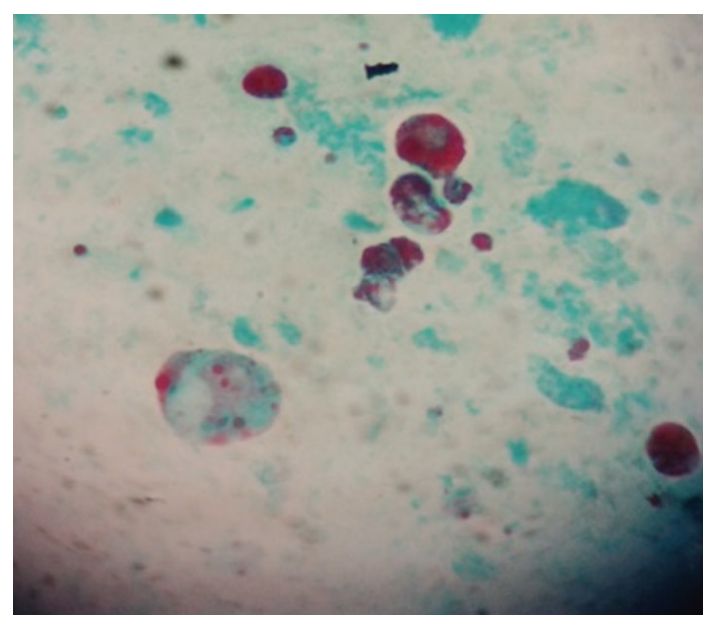

FIGURE 4: EH trophozoite (trichrome).

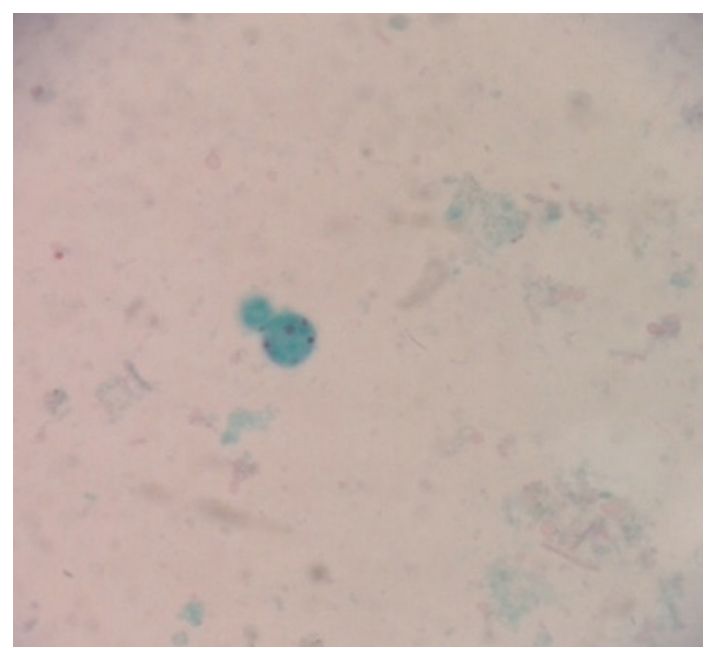

FIgURE 5: EH cyst (trichrome).

TABLE 2: Frequency of multiple organism infection.

\begin{tabular}{lc}
\hline Organism & Number \\
\hline E. histolytica + E. coli & $15(0.63 \%)$ \\
E. histolytica + G. lamblia & $2(0.08 \%)$ \\
E. coli + G. lamblia & $1(0.04 \%)$ \\
I. bütschlii + E. coli & $1(0.04 \%)$ \\
T. hominis + E. coli + I. bütschlii & $1(0.04 \%)$ \\
I. bütschlii + E. histolytica & $1(0.04 \%)$ \\
\hline
\end{tabular}

in trichrome and were 1-2 in number with blunt ends. Trophozoites, in saline mount, showed progressive motility with ingested debris in the cytoplasm. Nucleus and ingested material could not be identified. In iodine mount, irregularity of the cell outline with pseudopodia and single nucleus could be visualized. Trophozoites in trichrome stain (Figure 4)

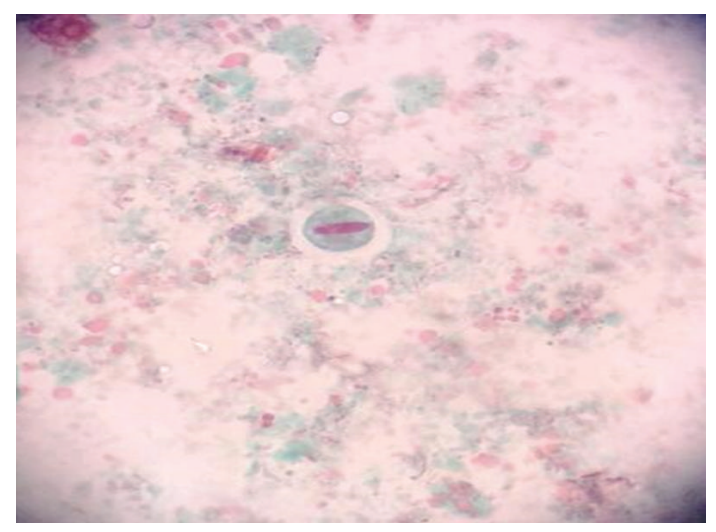

Figure 6: EH chromatoid body.

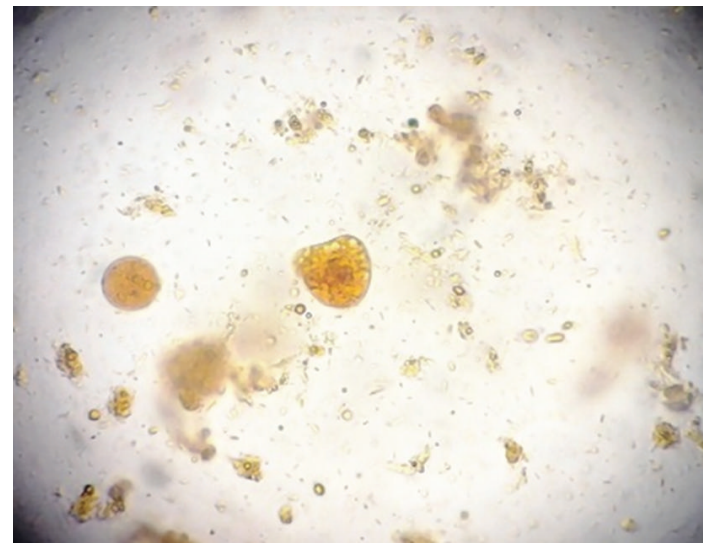

Figure 7: E. coli cyst (iodine).

TABLE 3: Organisms seen in diarrheal samples.

\begin{tabular}{lc}
\hline Organism & Number of diarrheal samples \\
\hline E. histolytica & 11 \\
E. coli & 11 \\
G. lamblia & 2 \\
E. histolytica + E. coli & 4 \\
T. hominis + E. coli + I. bütschlii & 1 \\
\hline
\end{tabular}

showed a ground glass cytoplasm with prominent red nucleus and ingested red blood cells.

E. coli. In saline, cysts of E. coli are seen as large spherical structures which may be easily mistaken for air bubbles. Only on lowering the condenser, hazy outlines of nuclei are made out. In iodine mount, (Figure 7) the nuclei stand out brilliantly against a brown cytoplasm. The nuclei in these cysts varied from 4 to 8 . However in trichrome stain, we found that the entire cell stains red and it can be overlooked as debris. Trophozoites are visualized in both saline and iodine mount due to their sluggish motility. However, presence of the nucleus with ingested debris is appreciated well in iodine 


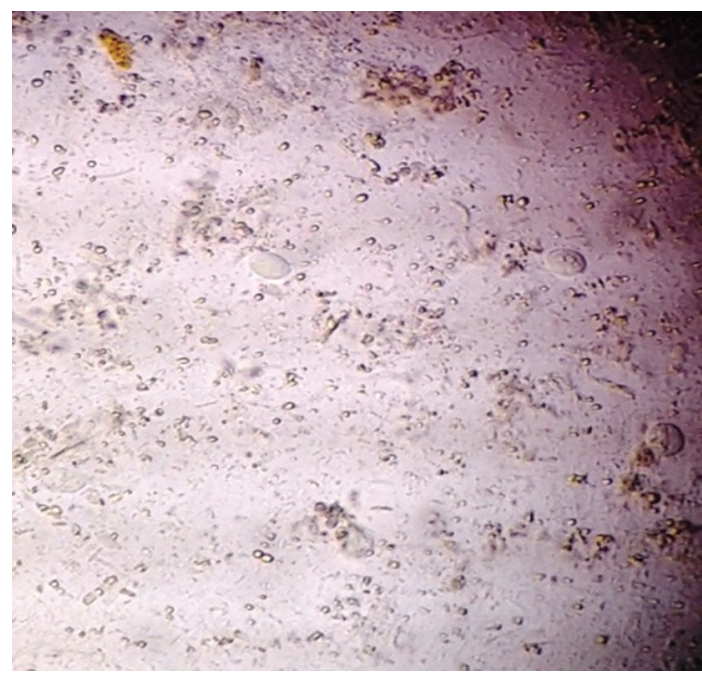

FIGURE 8: Giardia cyst (saline).

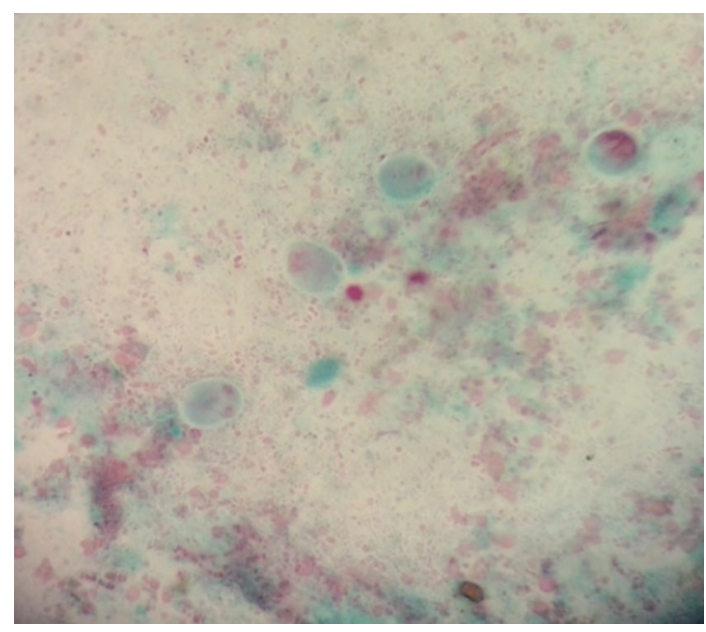

FIgURE 9: Giardia cyst (trichrome).

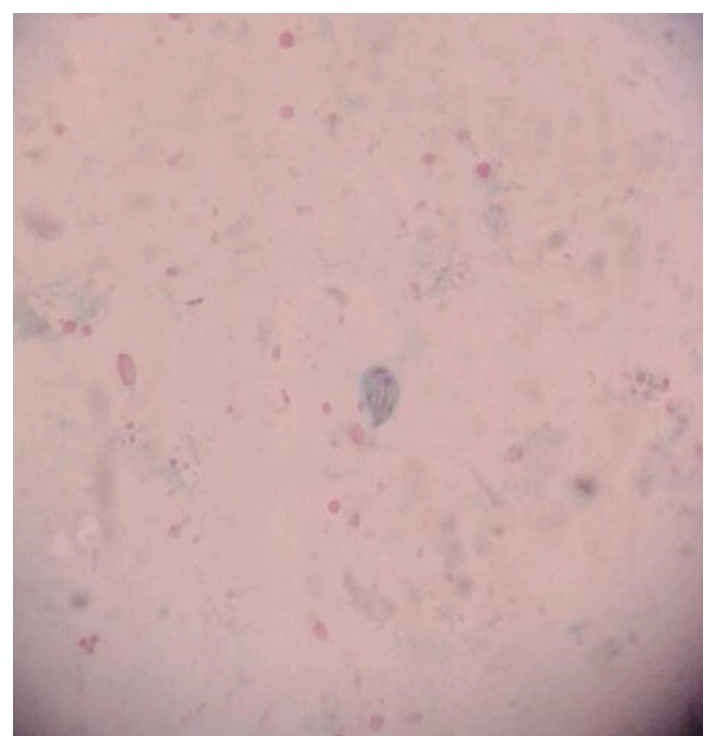

FIGURE 10: Giardia trophozoite (trichrome). mount. Trichrome did not highlight the trophozoites and entire cell appeared bright red.

G. lamblia. In saline mount (Figure 8), cysts appeared elliptical with few showing 2 small nuclei. Few of the cysts showed retraction of cytoplasm from cell wall. Iodine mount smears showed presence of the axostyle in addition to nucleus against a light brown cytoplasm. In trichrome stain (Figure 9) the median bodies were also seen. Trophozoites in saline and iodine mount showed a pear shape with falling leaf pattern motility. The nuclei and axostyle were seen well in iodine mount. In addition to these, flagella (stained red) were visualized in trichrome (Figure 10).

Iodamoeba bütschlii. We found cysts of I. bütschlii in 4 cases. In saline mount they were seen only as large, oval structures with a large vacuole. In iodine mount the glycogen vacuole is brilliantly highlighted. However, nuclei are not visualized. In trichrome stained smears (Figure 11), a single peripheral nucleus is visible along with a large unstained vacuole.

T. hominis. Trophozoites are well visualized in saline and iodine mount where they appear as small pear-shaped organisms with rapid jerky movement. In trichrome stain the outline of the organism is hazy and easily mistaken for debris.

C. mesnili. We observed trophozoites alone, in one case. In saline and iodine mount, they are large, pear shaped with single nucleus and a spiral groove with characteristic rotary movement. In trichrome stain, in addition we notice the anterior flagella.

E. vermicularis. We detected eggs of E. vermicularis (Figure 12) in 1 case. These planoconvex eggs with embryo inside were easily visualized in saline mount. They do not stain with trichrome.

S. stercoralis. Larvae were seen in 1 sample. In saline mount, the larvae were found to have rapid motility, short buccal canal, and prominent genital primordium. They do not stain with trichrome stain.

\section{Discussion and Conclusions}

In our study (Table 4) on 2355 stool samples we found parasites in $7.8 \%$ of which protozoa infection was the commonest $(7.8 \%)$ as was seen in other studies [3,5-9]. E. histolytica was found to be the most common protozoa in our study. This is similar to Srihari and Marothi's findings $[8,9]$. The commonest organisms involved in multiple organism infection were E. coli and E. histolytica. E. coli and E. histolytica were equally recovered from diarrheal samples.

Though Entamoeba histolytica cysts and trophozoites have been identified in wet mounts, we found that the morphological details were clearly visualized in trichrome stain whereas in saline at times it was difficult to differentiate from artifacts. E. coli was best appreciated in iodine mount. Giardia was visualized in wet mount and stained smears; however, the morphological details are clear in trichrome 
TABLE 4: Comparison with other studies (single organism infection).

\begin{tabular}{|c|c|c|c|c|c|c|c|c|}
\hline Study & $E H \%$ & Giardia \% & E. coli $\%$ & Chilomastix \% & Iodamoeba \% & Trichomonas \% & Pin worm \% & Strongyloides \% \\
\hline Saki & 1.3 & 4.5 & 9.1 & - & 5 & - & - & - \\
\hline Legesse & 12.7 & 6.2 & - & - & - & - & - & 5.8 \\
\hline Eligail & 0.14 & - & 4.08 & 0.41 & 1.79 & - & 0.02 & 0.07 \\
\hline Chandrashekhar & 1.7 & 13.2 & - & - & - & - & - & - \\
\hline Sehgal (women) & 4.6 & 6.9 & 11.5 & - & 1.1 & 2.3 & - & - \\
\hline Srihari & 43.8 & 10.5 & - & - & - & - & - & - \\
\hline Marothi & 10.5 & 3.9 & - & - & - & - & - & - \\
\hline Our study & 4.6 & 0.84 & 1.2 & 0.04 & 0.12 & 0.04 & 0.04 & 0.04 \\
\hline
\end{tabular}

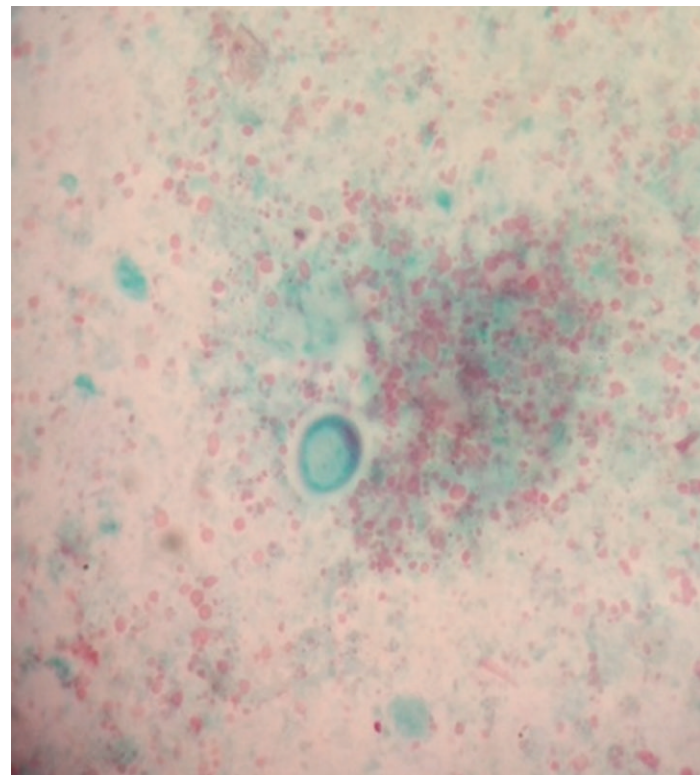

FIGURE 11: Iodamoeba cyst (trichrome).

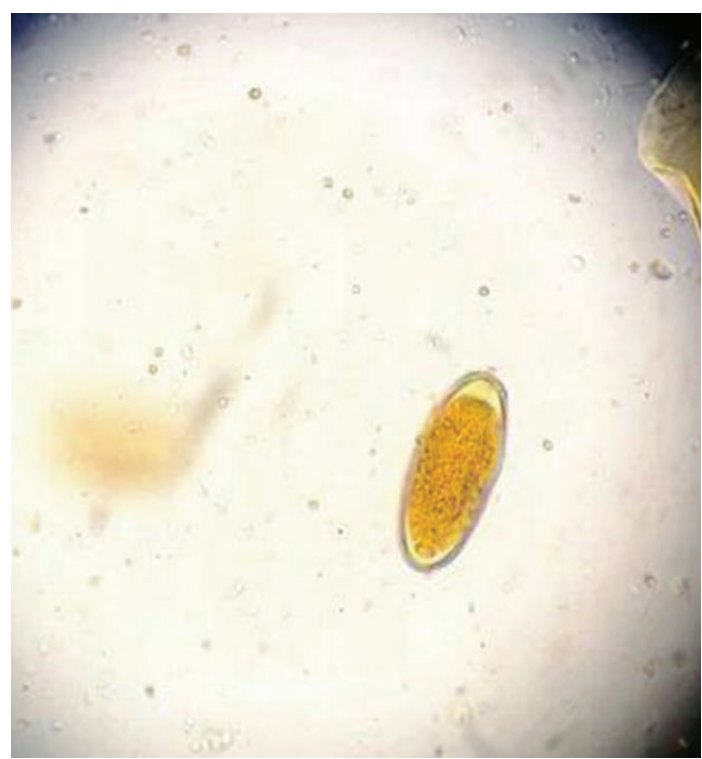

Figure 12: Pin worm (iodine). stain. Morphological identification of Iodamoeba is possible in iodine mount as well as trichrome stain. Morphological details of Trichomonas are not clear in wet mount or stained preparation. Its small size as well as jerky motility is helpful in diagnosis. Eggs of Enterobius and larvae of Strongyloides are seen in saline mount and neither the addition of iodine nor trichrome stain adds any benefit in diagnosis.

\section{Acknowledgments}

The authors would like to thank Ms Shali J., Ms Alice P., Ms Joice M.S., Ms Habibunissa A.G., Ms Ramya C., and Mr Saroj S. for their technical support.

\section{References}

[1] G. Kang, M. S. Mathew, D. P. Rajan et al., "Prevalence of intestinal parasites in rural Southern Indians," Tropical Medicine and International Health, vol. 3, no. 1, pp. 70-75, 1998.

[2] World health organization, Prevention and Control of Intestinal Parasitic Infection, vol. 749 of WHO Technical Reports Series, 1987.

[3] J. Saki, S. Khademvatan, K. Masoumi, and M. Chafghani, "Prevalence of intestinal parasitic infections among food handlers in Khuzestan, Southwest of Iran: a 10 year retrospective study," African Journal of Microbiology Research, vol. 6, pp. 2475-2480, 2010.

[4] M. Legesse and B. Erko, "Prevalence of intestinal parasites among school children in the rural area close to the southeast of Lake Langano," The Ethiopian Journal of Health Development, vol. 18, pp. 116-120, 2004.

[5] A. M. Eligail, A. M. Masawi, N. M. Al-Jaser, K. A. Abdelrahman, and A. H. Shah, "Audit of stool analysis results to ensure the prevalence of common types of intestinal parasites in Riyadh region, Saudi Arabia," Saudi Journal of Biological Sciences, vol. 17, no. 1, pp. 1-4, 2010.

[6] T. S. Chandrashekhar, H. S. Joshi, M. Gurung, S. H. Subba, M. S. Rana, and P. G. Shivananda, "Prevalence and distribution of intestinal parasitic infestation among school children in Kaski district, Western Nepal," Journal of Molecular Biology Research, vol. 4, pp. 78-82, 2005.

[7] R. Sehegal, G. V. Reddy, J. J. Verweij, and A. V. Rao, "Prevalence of intestinal parasitic infection among school children and pregnant women in a low socio economic area, Chandigarh, North India," Reviews in Infection, vol. 1, pp. 100-103, 2010.

[8] N. Srihari, T. S. Kumudini, J. Mariraj, and S. Krishna, "The prevalence of intestinal parasitic infection in a tertiary care 
hospital -a retrospective study," Journal of Pharmaceutical and Biomedical Sciences, vol. 12, pp. 1-4, 2011.

[9] Y. Marothi and B. Singh, "Prevalence of intestinal parasites at ujjain, Madhya Pradesh, India: five-year study," African Journal of Microbiology Research, vol. 5, no. 18, pp. 2711-2714, 2011. 


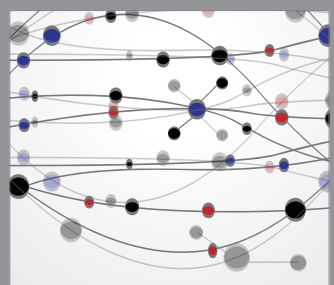

The Scientific World Journal
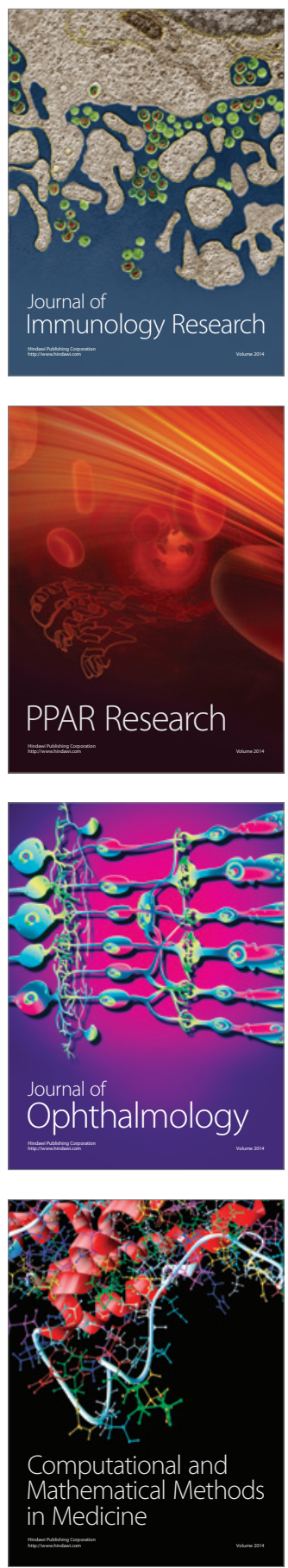

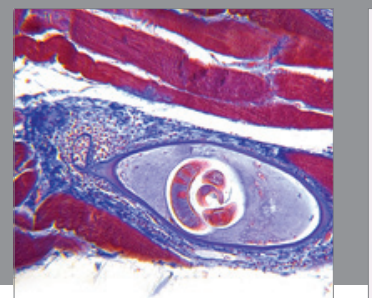

Gastroenterology

Research and Practice
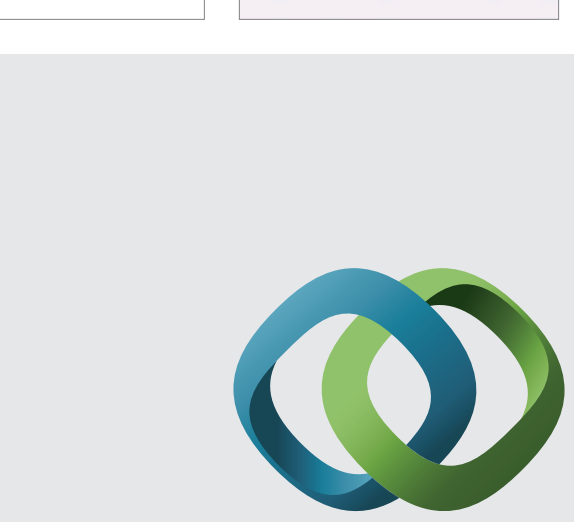

\section{Hindawi}

Submit your manuscripts at

http://www.hindawi.com
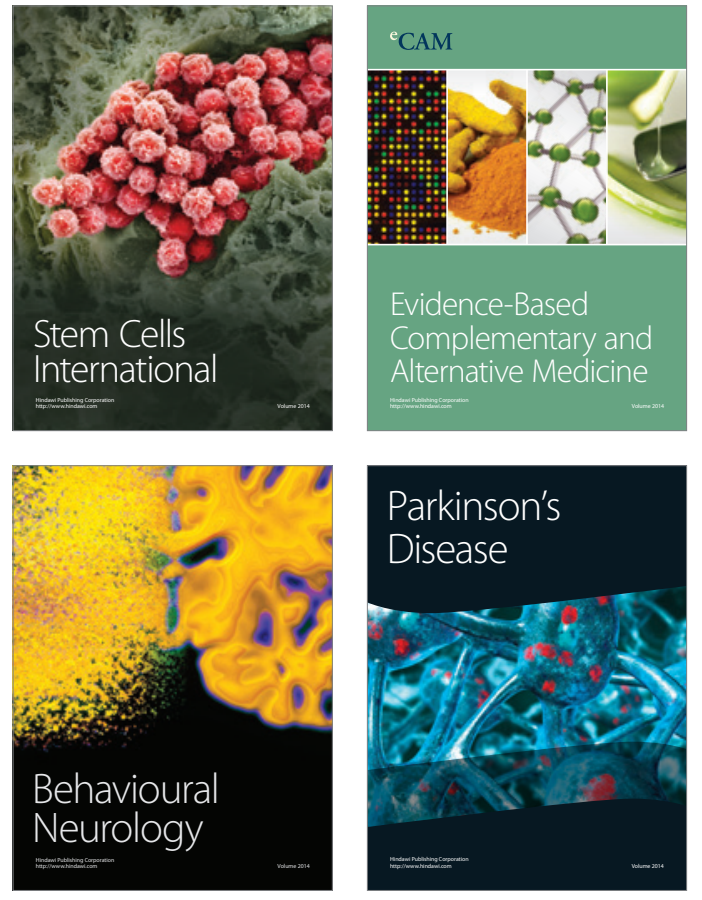
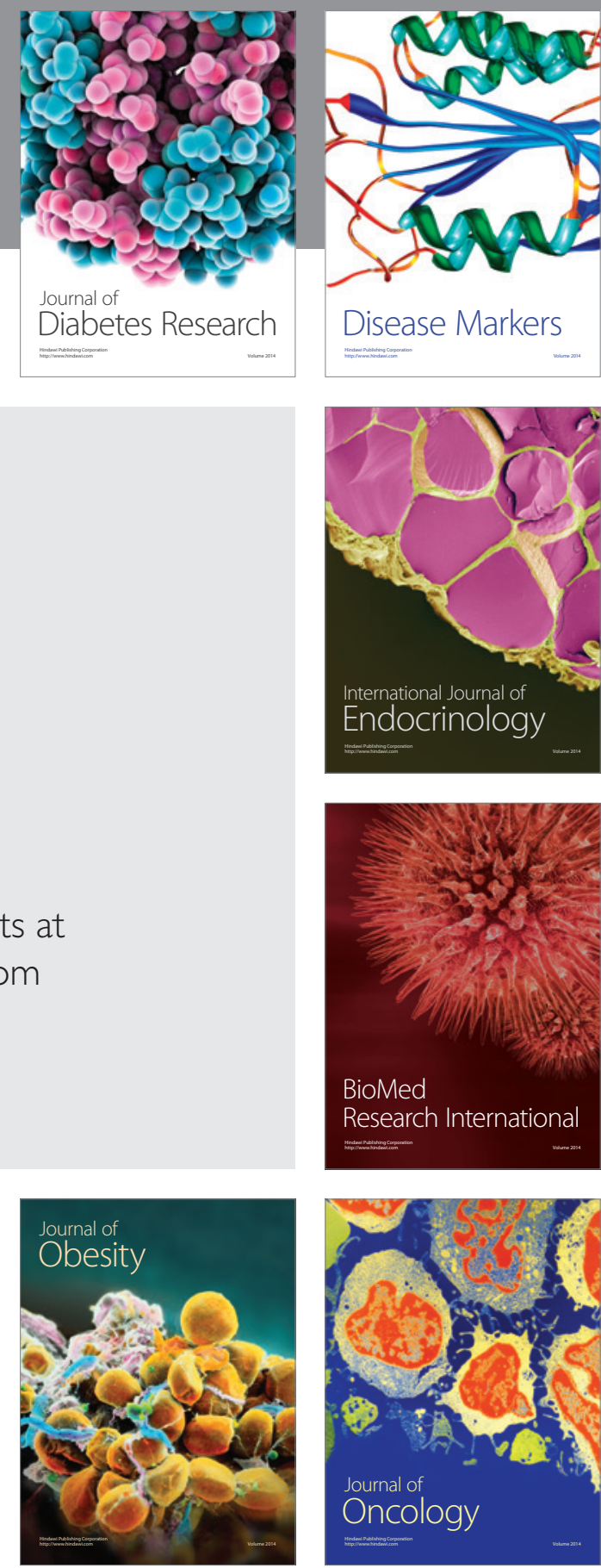

Disease Markers
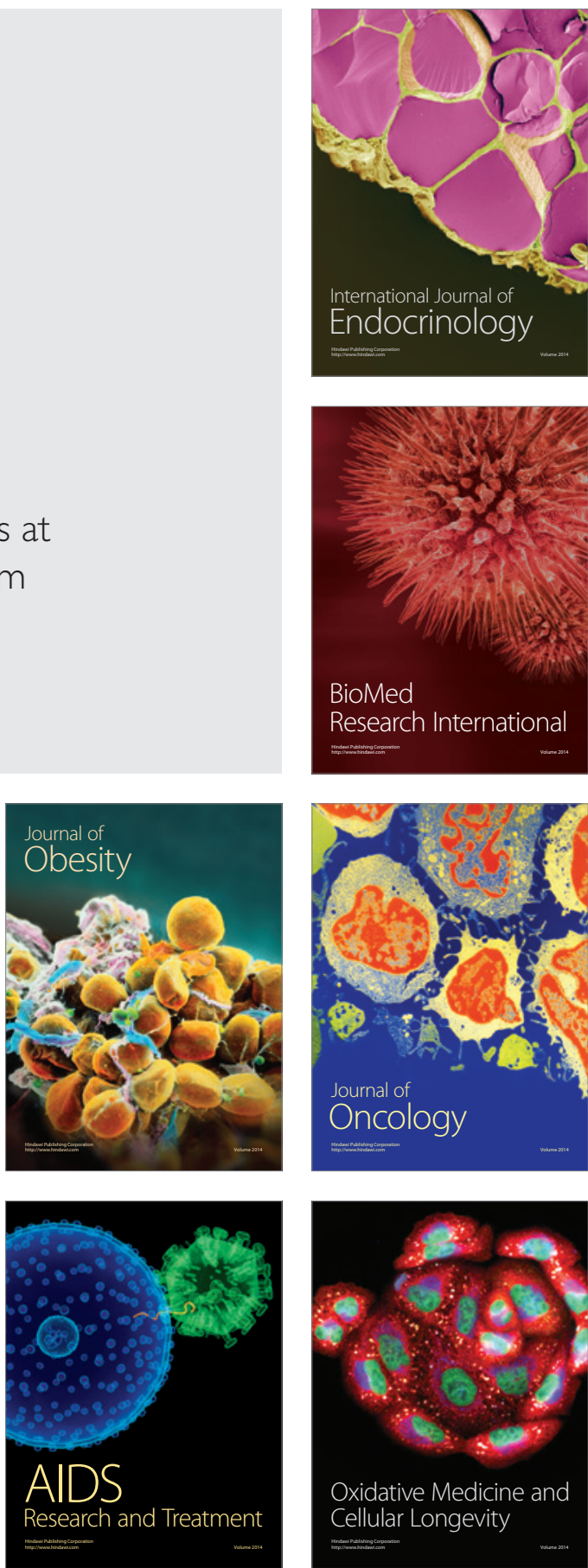